\title{
Cathepsin K Localizes to Equine Bone In Vivo and Inhibits Bone Marrow Stem and Progenitor Cells Differentiation In Vitro
}

\author{
Hussein $\mathrm{H}^{1}$, Boyaka $\mathrm{P}^{2}$, Dulin $\mathrm{J}^{1}$, Russell $\mathrm{D}^{2}$, Smanik L ${ }^{1}$, Azab $\mathrm{M}^{1}$, Bertone $\mathrm{AL}^{1,2}$
}

\begin{abstract}
Selective inhibition of Cathepsin K (CatK) has a promising therapeutic potential for diseases associated with bone loss and osseous inflammation, such as osteoarthritis, periodontitis, and osteoporosis. In horses, stress-related bone injuries are common and accompanied by bone pain and inflammation resulting in excessive bone resorption and periostitis. VEL-0230 is a highly selective inhibitor of CatK that significantly decreased bone resorption and increased bone formation biomarkers. The goal of this study was to demonstrate the presence of CatK in equine bone and a simultaneous influence on the bone marrow cellular components including function and differentiation. Our objectives were: 1) to investigate the tissue localization of CatK protein in equine bone using immunohistochemistry, and 2) to determine the effect of CatK inhibition on osteoclastogenic, chondrogenic and osteogenic differentiation potential of equine stem and progenitor cells in vitro using histochemical staining and differentiation-related gene expression analyses. Bone biopsies, harvested from the tuber coxae and proximal phalanx of six healthy horses, were processed for immunostaining against CatK. Sternal bone marrow aspirates were cultured in $0,1,10$, or $100 \mu \mathrm{M}$ of VEL-0230 and subsequent staining scoring and gene expression analyses performed. All cells morphologically characterized as osteoclasts and moderate number of active bone lining osteoblasts stained positive for CatK. Histochemical staining and gene expression analyses revealed a significant increase in the osteoclastogenic, chondrogenic and osteogenic differentiation potential of equine bone marrow cells, which was VEL-0230-concentration dependent for the latter two. These results suggested that CatK inhibition may have anabolic effects on bone and cartilage regeneration that may be explained as a feedback response to CatK depletion. In conclusion, the use of CatK inhibition to reduce inflammation and associated bone resorption in equine osseous disorders may offer advantages to other therapeutics that would require further study.
\end{abstract}

Key Words: Osteoblast, Osteoclast, Chondrocyte, Osteoporosis, Bone resorption

\section{Introduction}

Cathepsin K (CatK) has become a major therapeutic target for the treatment of bone loss from osteoporosis ${ }^{[1]}$, with potential for use in other diseases associated with bone resorption. The discovery of CatK, the predominant collagenase expressed in osteoclasts (OC), has revealed the significance of its inhibition of bone resorption via suppression of osteoclast activity. Multiple CatK inhibitors have been developed and have shown variable success in clinical trials to increase bone density and decrease pain ${ }^{[2-4]}$. Inhibitors of CatK, such as Odanacatib and VEL-0230/NC-2300, block the entire activity of CatK and can interfere with other signaling pathways known to be under the influence of CatK such as the innate immune response and inflammation ${ }^{[4-7]}$. We have investigated the pharmacokinetics of a potent CatK inhibitor, VEL-0230, in healthy exercising horses for future therapeutic application in equine patients with osteo-inflammatory conditions $^{[8]}$. Cathepsin $\mathrm{K}$ inhibition ameliorated the inflammatory response of equine bone marrow mononuclear cells stimulated by Toll like receptors (TLR) -4 and TLR-9 ligands in vitro along with evidence of suppressed bone resorption and increased bone formation in vivo due to repeated VEL-0230 administration in healthy exercising horses ${ }^{[9,10]}$. The precise role of CatK in the immune system is unclear and maybe attributed, in part, to the location and tight integration between the bone and bone marrow. The latter constitutes the body reserve for immune, stem and progenitor cells.

Significant levels of CatK expression have been detected in osteoclasts, synovium, and chondrocytes of human and animal tissues $^{[11-14]}$. One study has also demonstrated significant CatK expression in osteocytes and osteoblasts of human decalcified bone sections ${ }^{[15]}$. Similarly, expression levels of CatK were detected in equine ex-vivo cartilage tissue and in osteoclast-like cells generated in vitro ${ }^{[16,17]}$. However, CatK expression pattern in equine bone has not been studied, and may be relevant to equine bone and inflammatory diseases. Equine bone marrow serves as a source of mononuclear cells, growth factors and stem and progenitor cells. It has been used clinically to promote repair of musculoskeletal tissues including ligaments, tendons and articular cartilage regeneration ${ }^{[18-20]}$. Stem and progenitor cells differentiation has been found to share many pathways and common molecular regulatory factors with the immune system ${ }^{[21-24]}$. Hence, we hypothesized that CatK inhibition in vitro, using VEL-0230 in concentrations similar to the ones which suppressed inflammation in stimulated equine bone marrow nucleated cells ${ }^{[9]}$, would significantly alter the

Author Names in full: Hayam Hussein ${ }^{1}$, Prosper Boyaka ${ }^{2}$, Jennifer Dulin ${ }^{1}$, Duncan Russell², Lauren Smanik ${ }^{1}$, Mohamed Azab ${ }^{1}$, Alicia L. Bertone ${ }^{1,2}$

${ }^{1}$ Department of Veterinary Clinical Sciences, College of Veterinary Medicine, The Ohio State University, Columbus, OH, USA; ${ }^{2}$ Department of Veterinary Biosciences, College of Veterinary Medicine, The Ohio State University, Columbus, OH, USA. 
differentiation potential of equine stem and progenitor cells along the three main cell lineage of the skeletal system, which may provide relevance into the effect of VEL-0230 on bone. Our study aimed, firstly, to investigate the tissue localization of CatK protein in equine bone using immunohistochemistry and subsequent histomorphologic analysis. The second aim of this study was to determine the effect of CatK inhibition on osteoclastogenic, chondrogenic, and osteogenic differentiation in vitro using histochemical stains and differentiationrelated gene expression analyses. For the second aim, osteoclastogenic, chondrogenic, and osteogenic induced cells were processed for tartrate-resistant acid phosphatase (TRAP), toluidine blue, von Kossa and alizarin red $\mathrm{S}$ staining, respectively and subsequent staining scoring was performed. Furthermore, quantitative real time polymerase chain reaction (qRT-PCR) was performed to quantify relative gene expression associated with osteoclastogenic differentiation (CatK, Receptor activator of nuclear factor kappa-B ligand [RANKL], TRAP), chondrogenic differentiation (Aggrecan, Collagen 2A, SOX9), and osteogenic differentiation (alkaline phosphatase, osteopontin, Runt-related transcription factor 2 [RUNX2]).

\section{Materials And Methods}

\subsection{Animals}

Seven young horses (3-5 years), three females and four males, free from lameness and systemic disease based on physical examination, were used in this study. All experimental protocols were pre-approved by the Institutional Animal Care and Use Committee (IACUC) of the Ohio State University.

\subsection{Bone biopsy}

Biopsy samples were collected from six of the horses from the Tuber coxae (TC), a non-weight-bearing bone, and the proximal phalanx (P1), a weight-bearing bone, as previously described ${ }^{[10]}$. Horses were randomly assigned to left or right TC and forelimb $\mathrm{P} 1$.

\subsection{Immunostaining of bone CatK}

Cathepsin $\mathrm{K}$ was immunostained in representative sections of formalin-fixed decalcified $\mathrm{TC}$ and $\mathrm{P} 1$ bone specimens using a standard Biotin/streptavidin horseradish peroxidase method ${ }^{[25]}$. Paraffin sections were de-waxed through Xylene (Sigma-Aldrich, St. Louis, MO) and descending gradient of ethanol treatments. To reduce nonspecific binding, the tissue samples were treated with the avidin/biotin blocking kit per manufacturer's instructions (SP-2001, Vector Laboratories, Burlingame, CA), followed by three 5 minute washes with Phosphate buffered Saline (PBS). Bone CatK was then immunostained using a pre-validated Rabbit polyclonal CatK antibody (1:50) as a primary antibody (Biorbyt Ltd, Cambridgeshire, UK) with which samples were incubated at $4{ }^{\circ} \mathrm{C}$ overnight followed by incubation for 30 minutes with biotinylated Goat anti-Rabbit immunoglobulins (Vector Laboratories, UK) as a secondary antibody. Bone samples were developed with a peroxidase substrate solution (NovaRED, Vector Laboratories, Burlingame, CA) for 10 minutes to stain CatK-positive osteoclasts. Slides were washed quickly in PBS to terminate the reaction, taped dry, counterstained with hematoxylin, taped dry, cover-slipped, and allowed to dry in the dark for 2 days. Three regions of interest (ROIs) within the trabecular bone of TC and P1 were analyzed under 200x magnification for determination of total number of osteoclasts, total number of bone lining osteoblasts, CatKpositive osteoclasts, CatK-positive bone lining (endosteal) osteoblasts and bone surface (BS) using Bioquant OSTEO software (Bioquant OSTEO 7.20.10; Bioquant Image Analysis Co.). The staining intensity of CatK-positive osteoclasts and osteoblasts was scored by a blinded evaluator $(\mathrm{HH})$ as following: 0 (no staining), 1 (mild staining), 2 (moderate staining), 3 (marked staining). The average percentage of CatK-positive osteoclasts was calculated as (CatKpositive OC/total OC). In addition the average number of CatKpositive OC were quantified per bone surface (N.OC/mmBS).

\subsection{Bone marrow aspiration and processing for cell differentiation assays}

Sternal bone marrow was isolated from seven horses and processed according to the methods previously described ${ }^{[9]}$. The processed bone marrow concentrates from each horse was cultured in monolayer under four experimental conditions, in triplicate, of $0,1,10$, or $100 \mu \mathrm{M}$ of a CatK inhibitor (VEL-0230) (Freestride Therapeutics Inc., MI, USA) in culture media, to select both the adherent cells (bone marrow derived-mesenchymal stromal cells (BMD-MSCs) which were driven down the chondrogenic and osteogenic pathways) or the non-adherent fraction which was driven down the osteoclastogenic pathway.

\subsubsection{Osteoclastogenic differentiation and staining assay}

An aliquot of fresh concentrated bone marrow was cultured for six hours in $10 \%$ FBS supplemented DMEM, at approximately $1 \times 10^{7}$ cells $/ \mathrm{cm}^{2}$ in a $75 \mathrm{~cm}^{2}$ culture flask, $37^{\circ} \mathrm{C}, 5 \% \mathrm{CO}_{2}$. The nonadherent cells were driven to differentiate into equine osteoclast-like cells (OCLs); separated by centrifugation at $500 \mathrm{~g}, 4^{\circ} \mathrm{C}$ for 10 minutes and re-suspended in $\alpha$-MEM, $\mathrm{pH} 7.2$, supplemented with 10 $\% \quad(\mathrm{v} / \mathrm{v})$ FBS, 25 $\mathrm{ngml}^{-1}$ rhM-CSF (R\&D Systems), 10ngml ${ }^{-1}$ rhRANKL (R\&D Systems), 10 ${ }^{-8}$ M 1,25 dihydroxyvitamin D3 (vitamin D3, Affiniti Research Products, UK). Cells were plated at a density of $2.5 \times 10^{6}$ cells $\mathrm{cm}^{2}$ in collagen-coated six well plates. Half the medium was replaced twice weekly. After 7 days, non-adherent cells were removed by trypsinization for 2 minutes. After 15 minutes of further trypsinization the remaining cells, which were enriched for OCLs, were collected by gentle scraping using a cell scraper and cytospins on poly-Llysine coated slides (Sigma, St Louis, MO) were performed. The cytospin preparations of OCLs were processed for TRAP staining using a commercial acid phosphatase leucocyte kit (Sigma, St Louis, MO) according to the manufacturer's protocol. The number of TRAP-positive multinucleated cells ( $\geq 3$ nuclei) in five representative fields under $200 \mathrm{X}$ magnification was counted and averaged to determine the representative number of OCLs for each experimental condition.

\subsubsection{Chondrogenic differentiation and staining assay}

Bone marrow-derived MSCs, selected by adherence in monolayer culture of processed fresh bone marrow concentrates for up to 3 days, were isolated and differentiated to chondrocytes using Lonza ${ }^{\circledR}$ Poietics TM human mesenchymal stem cell media and modified protocol. Fifteen $\mathrm{ml}$ centrifuge tubes were used for chondrogenic induction, with $1 \times 10^{6} \mathrm{MSCs} /$ tube to form a pellet. After induction for 21 days, pellets were removed and fixed by incubation in formalin for 24 hours. Formalin-fixed chondrocyte pellets were sectioned and stained for glycosaminoglycan with toluidine blue stain (Fisher scientific, USA) for 30 seconds and then washed briefly in tap water. The degree of cartilaginous extracellular matrix staining (purple color) was scored by a blinded evaluator $(\mathrm{HH})$ in three microscopic fields under $200 \mathrm{X}$ magnification as: 0 (no staining), 1 (mild staining; < $30 \%$ of the microscopic field), 2 (moderate staining; 30-60\%), 3 (marked staining; > 60\%).

\subsubsection{Osteogenic differentiation and staining assay}

A fraction of the BMD-MSCs were differentiated to osteoblasts using Lonza ${ }^{\circledR}$ Poietics TM human mesenchymal stem cell media and modified protocol. A 6-well plate was used for osteogenic induction with $1 \times 10^{6} \mathrm{MSCs} /$ well. Basal medium was used for the first 24 hours to allow adherence. Induction medium was changed every 
3 days thereafter. The cells were analyzed after 28 days for deposition of calcium phosphate and calcium, in accordance with a von Kossa and alizarin red S staining protocols, respectively. In brief, the wells were washed twice with PBS solution and then fixed in phosphatebuffered $10 \%$ formalin for 10 minutes. The monolayers were then processed for von Kossa and alizarin red S staining. For von Kossa staining, cells were serially dehydrated in solutions of ethanol $(70 \%$, $95 \%$, and $100 \% ; 2$ times for each concentration) and then allowed to air dry. Cells were then serially rehydrated in solutions of ethanol $(100 \%, 95 \%$, and $80 \% ; 2$ times for each concentration). Finally, cells were rehydrated in water twice. Silver nitrate solution (2\%) was added, and the cells were exposed to sunlight for 20 to 40 minutes. The cells were then rinsed 2 times with water, and 5\% sodium thiosulfate was added; cells were allowed to equilibrate for 3 minutes. Cells then were rinsed with water, and acid fuchsin counterstain (5 $\mathrm{mL}$ of $1 \%$ acid fuchsin with $95 \mathrm{~mL}$ of picric acid and $0.25 \mathrm{~mL}$ of $12 \mathrm{~N}$ $\mathrm{HCl})$ was added. After incubation for 5 minutes, the cells were washed with water (2 times), $95 \%$ ethanol, and $100 \%$ ethanol before image analysis by use of a viewing microscope where mineralized nodules were stained dark brown or black. For Alizarin red $\mathrm{S}$ staining, 2\% alizarin red S (Sigma) was prepared in distilled water and the $\mathrm{pH}$ was adjusted to 4.1-4.3 using 0.5\% ammonium hydroxide. Formalin-fixed osteogenic monolayers were washed, and stained with alizarin red $\mathrm{S}$ for $10-15$ minutes. After removal of unincorporated excess dye with distilled water, the mineralized nodules were labeled as red spots. The degree of mineralized nodules staining with each stain was scored by a blinded evaluator $(\mathrm{HH})$ in three microscopic fields under 20X magnification as following: 0 (no staining), 1 (mild staining; < $30 \%$ of the microscopic field), 2 (moderate staining; 30-60\%), 3 (marked staining; > 60\%).

\subsection{Gene expression analyses}

Total RNA was isolated using Guanidinium thiocyanate-phenolchloroform extraction method (TRIzol ${ }^{\circledR}$ and Chlorophorm;
Invitrogen). Complimentary (c) DNA was generated from $1 \mu \mathrm{g}$ total RNA using High Capacity cDNA Reverse Transcription Kits (Applied Biosystems, Foster City, CA, USA) according to the manufacturer's instructions using MJ Research PTC-150 Thermal Cycler (MJ Research, USA). Relative gene expression analysis was performed using equine-specific custom primers (Primer3 software; Steve Rozen, Helen J. Skaletsky, 1998). Quantification of gene expression relevant to the osteoclastogenic, chondrogenic and osteogenic pathways using qRT-PCR was performed for all experimental conditions of the differentiation assays in a total volume of $50 \mu \mathrm{l}$ using 96-well microwell plates, an ABI PRISM 7000 sequence detector and SYBR® Green PCR Master Mix (Applied BioSystems). Cycle threshold $(\mathrm{Ct})$ values were obtained for all analyzed genes during log phase of the cycle and expression level was compared between different experimental conditions using 2- $-\Delta \Delta$ Ct. Non-induced cells were used as reference samples and Glyceraldehyde 3-phosphate dehydrogenase (GAPDH) was used as a reference housekeeping gene. Table (1) lists the corresponding forward and reverse nucleotides sequences for the primers used in the qRT-PCR.

\subsection{Statistical analysis}

The data was graphed as mean with standard deviation (SD), with the exception of the chondrogenic and osteogenic staining score data, which was graphed as median and interquartile range. All data were statistically analyzed using SPSS v.18.0 (IBM Corp., Armonk, NY, USA). Normality within the different variables was investigated using Shapiro-Wilk test. The comparison between the TC and P1 data was performed using paired sample student $t$ test. Comparisons between different CatK inhibitor concentrations were performed using Friedman test for the chondrogenic and osteogenic staining scored data, and the one-way analysis of variance with repeated measures for all other data, followed by Duncan's Multiple Range Test. Significance was determined when $\mathrm{P}<0.05$.

Table 1: The forward and reverse nucleotides sequences for primers used in the q RT-PCR.

\begin{tabular}{|c|c|c|}
\hline & Gene & Primer sequences \\
\hline \multirow{3}{*}{$\begin{array}{c}\text { Osteoclastogenic } \\
\text { differentiation-related } \\
\text { genes }\end{array}$} & CatK & $\begin{array}{l}\text { Fwd: 5'CAGAGATTGCCATTCCGTTT3' } \\
\text { Rev: 5'ATAGAATCAGCCCCAGGACA3' }\end{array}$ \\
\hline & RANKL & $\begin{array}{l}\text { Fwd: 5'CGACATCCCATCAGGTTCCC3' } \\
\text { Rev: 5' CCCGACCAGTATTTGGTGCT3' }\end{array}$ \\
\hline & TRAP & $\begin{array}{l}\text { Fwd: 5'CTGGTCTTGAACAGGGACTTG3' } \\
\text { Rev: 5'AGAACGGCATAGGCTTTGTG3' }\end{array}$ \\
\hline \multirow{3}{*}{$\begin{array}{c}\text { Chondrogenic } \\
\text { differentiation-related } \\
\text { genes }\end{array}$} & Aggrecan & $\begin{array}{l}\text { Fwd: 5'TGCACAGACCCCGCCAGCTA3' } \\
\text { Rev: 5'GTCTCTAAACTCAGTCCACG3' }\end{array}$ \\
\hline & Collagen 2A & $\begin{array}{l}\text { Fwd: 5' GCTTCCACTTCAGCTATGGA3' } \\
\text { Rev: 5’ TGTTTCGTGCAGCCATCCTT3' }\end{array}$ \\
\hline & SOX9 & $\begin{array}{l}\text { Fwd: 5'CGCCGAAGCTCAGCAAGA3' } \\
\text { Rev: 5'CGCTTCTCGCTCTCGTTCA3' }\end{array}$ \\
\hline \multirow{3}{*}{$\begin{array}{c}\text { Osteogenic } \\
\text { differentiation-related } \\
\text { genes }\end{array}$} & Alkaline phosphatase & $\begin{array}{l}\text { Fwd: 5'TGGGGCTAAGCTCTGGAGTC3' } \\
\text { Rev: 5'ACTGATGTTCCAATCCTGCTCG3' }\end{array}$ \\
\hline & Osteopontin & $\begin{array}{l}\text { Fwd: 5'CGCAGATCTGAAGACCAGTA3' } \\
\text { Rev: 5'GGAATGCTCACTGGTCTCAT3' }\end{array}$ \\
\hline & RUNX2 & $\begin{array}{l}\text { Fwd: 5'CTCCAACCCACGAATGCACTA3' } \\
\text { Rev: 5'CGGACATACCGAGGGACATG3' }\end{array}$ \\
\hline Internal control gene & GAPDH & $\begin{array}{l}\text { Fwd: 5'CAACGAATTTGGCTACAGCA3' } \\
\text { Rev: 5'CTGTGAGGAGGGGAGATTCA3' }\end{array}$ \\
\hline
\end{tabular}




\section{Results}

\subsection{CatK protein distribution in equine decalcified bone sections}

All morphologically characterized osteoclasts stained positive for CatK in both TC and P1 trabecular bone and there was no difference in CatK staining intensity between the two bone types (data not shown). Figure 1A shows a microscopic image of Cathepsin Kpositive, multinucleated osteoclasts within the resorption pits. There was a significant increase in the number of CatK-positive osteoclasts per bone surface in the P1 compared to the TC trabecular bone data (Figure 1B). A moderate number of active bone lining osteoblasts stained CatK-positive. The percent of CatK-positive osteoblasts did not differ between trabecular bone of $\mathrm{TC}$ and $\mathrm{P} 1$, and were $65.4 \pm 7.4 \%$ and $62.6 \pm 6.2 \%$ of total bone lining osteoblasts, respectively. Figure $1 \mathrm{C}$ shows a microscopic image of CatK-positive osteoblasts. The staining intensity of CatK-positive osteoblasts did not differ between both bone types (data not shown).

\subsection{Effect of CatK inhibition on osteoclastogenic potential of equine progenitor cells}

The osteoclastogenic-induced cells of all four experimental conditions showed had varying numbers of TRAP-positive multinucleated OCLs (Figure 2A). There was significant increase in the number of TRAP-positive multinucleated OCLs due to CatK inhibition at VEL-0230 concentration of $100 \mu \mathrm{M}$ compared to the other 3 experimental conditions (Figure 2B). Gene expression analysis revealed increases in relative CatK and RANKL gene expression at $100 \mu \mathrm{M}$ concentration, and in relative TRAP gene expression at 10 and $100 \mu \mathrm{M}$ concentration compared to the 0 concentration of VEL-0230 in culture media (Figure 2C).

\subsection{Effect of CatK inhibition on chondrogenic potential of equine BMD-MSCs}

The toluidine blue-stained sections of chondrogenic pellets showed production of glycosaminoglycan from chondrocytes (Figure 3A). There was a significant increase in toluidine blue staining score due to CatK inhibition at VEL-0230 concentration of $100 \mu \mathrm{M}(\mathrm{P}<0.05)$, compared to the other concentrations (Figure 3B). There were significant increases in relative Aggrecan, Collagen 2A and SOX9 gene expressions $(\mathrm{P}<0.05)$, which were VEL-0230- concentration dependent (Figure 3C).

\subsection{Effect of CatK inhibition on osteogenic potential of equine BMD- MSCs}

The induced BMD-MSCs formed osteoblasts were stained positive for von Kossa (Figure 4A) and Alizarin Red-S (Figure 4B). There was a significant increase in both von Kossa and Alizarin Red-S staining scores due to CatK inhibition at a VEL-0230 concentration of 10 and $100 \mu \mathrm{M}(\mathrm{P}<0.05)$, compared to the other experimental conditions (Figure 4C). There were significant increases in relative alkaline phosphatase, osteopontin, and RUNX2 gene expressions $(\mathrm{P}<0.05)$, which were VEL-0230- concentration dependent (Figure 4D).
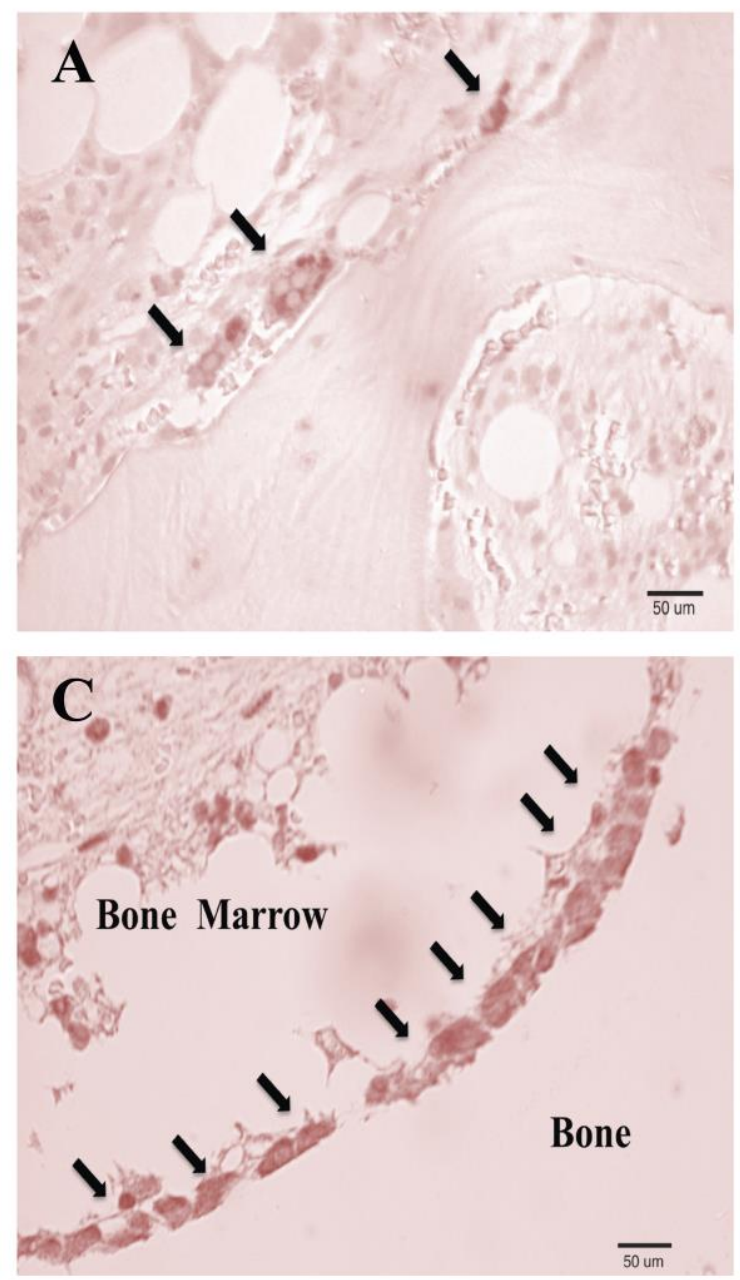

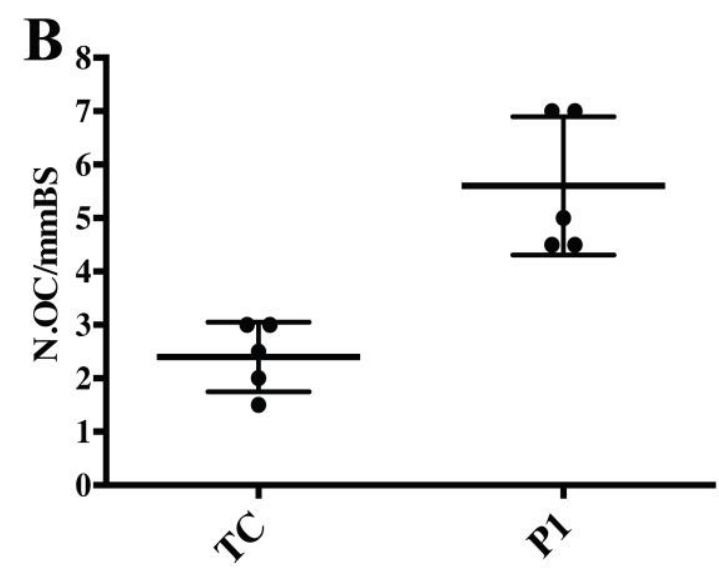

Trabecular bone origin
Figure 1 Cathepsin $K$ (CatK) protein distribution in equine decalcified bone sections. There were no differences between tuber coxae (TC) and first phalanx (PI) trabecular bone percent and staining intensity of CatK-positive osteoclasts $(O C)$ and osteoblasts. A. A microscopic image of Cathepsin K-positive, multinucleated osteoclasts (black arrows) within the resorption pits in one region of interest. $\boldsymbol{B}$. Mean \pm standard deviation of CatK-positive OC per bone surface, showing significant increase in number of CatK-positive $O C$ in trabecular bone of $P 1$ compared to TC $(P<0.05)$. C. A microscopic image of CatKpositive osteoblasts (black arrows) in one region of interest. 
A
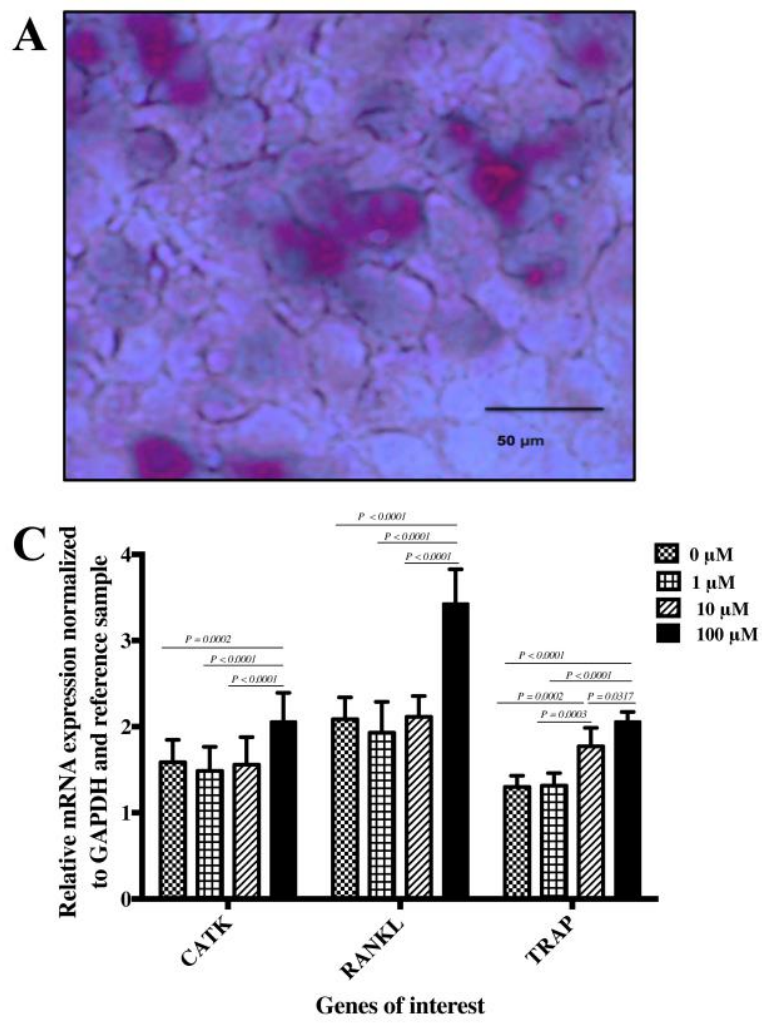

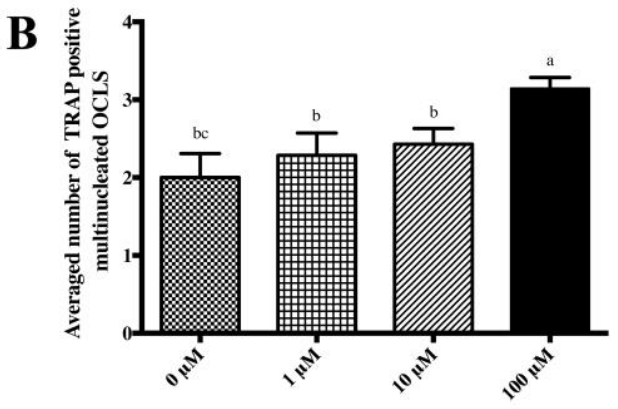

Cathepsin $\mathrm{K}$ inhibitor concentrations

Figure 2 Effect of CatK inhibition on osteoclastogenic potential of equine progenitor cells. A. A microscopic image of bone marrow derived-mononuclear fraction cultured in osteoclastogenic media showing marked TRAP positive multinucleated osteoclast-like cells $(O C L S)$. B. Mean \pm standard deviation of averaged number of TRAPpositive OCLs; there was a significant increase due to CatK inhibition at a concentration of $100 \mu M$ compared to the other concentrations $(P<0.05)$. Different letters refer to the significance between different concentrations within the same gene data. $C$. Analysis of gene expression relevant to the osteoclastogenic pathway (CatK, RANKL and TRAP), using quantitative real time polymerase chain reaction ( $q$ $R T-P C R)$, under different concentrations of a CatK inhibitor, normalized to GAPDH and reference sample. There were significant increases in relative CatK and RANKL gene expression at $100 \mathrm{uM}$ concentration $(P<0.05)$, and in relative TRAP gene expression at 10 and $100 \mathrm{uM}$ concentration compared to the 0 concentration of VEL-0230 in culture.
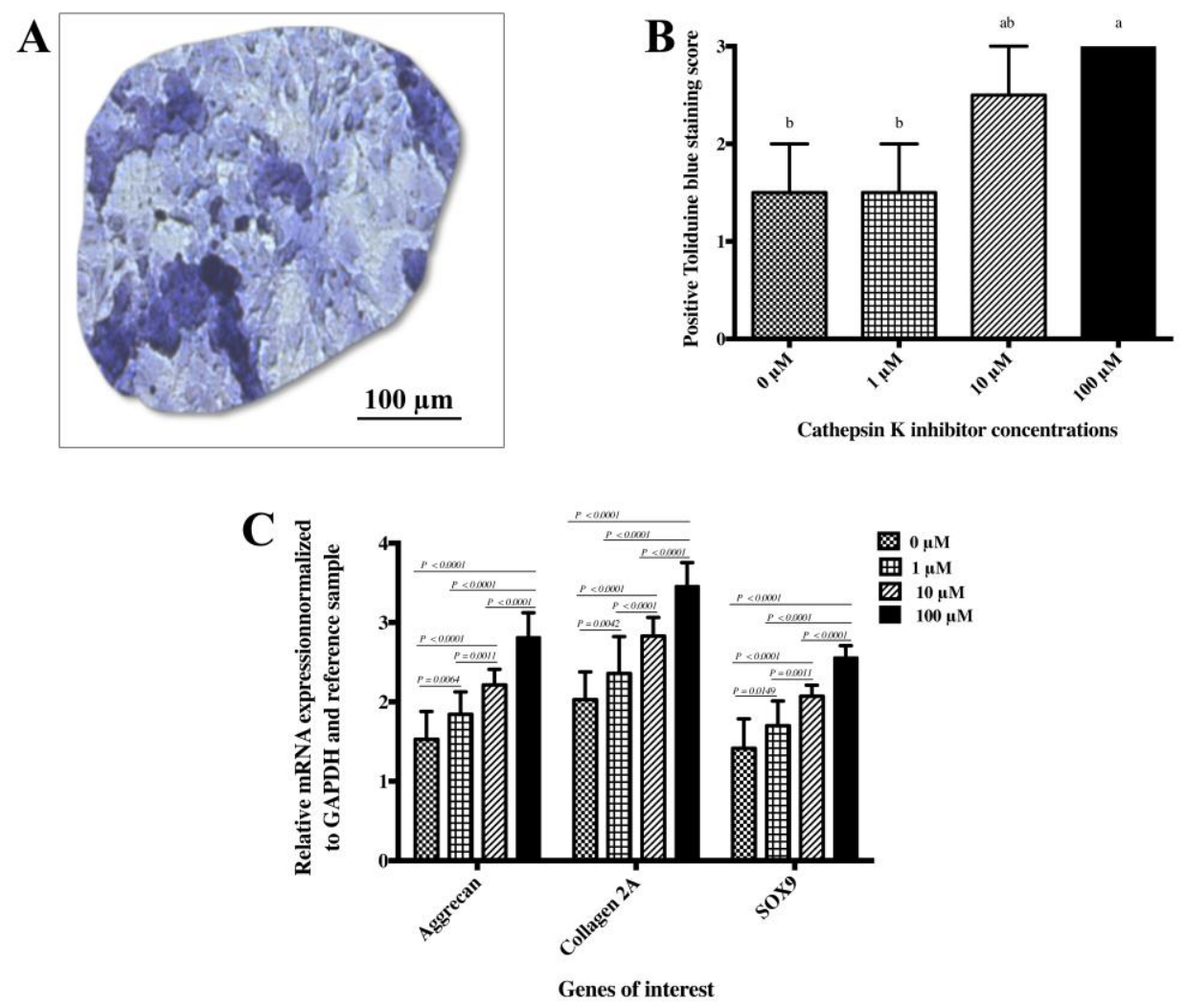

Figure 3 Effect of CatK inhibition on chondrogenic potential of equine bone marrow-derived mesenchymal stromal cells (BMD-MSCs). A. A microscopic image of toluidine blue staining of a chondrogenic pellet. B. Median with interquartile range of toluidine blue staining score of chondrogenic pellets; there was a significant increase due to CatK inhibition at a concentration of $100 \mu \mathrm{M}$ compared to the other concentrations $(P<0.05)$. Different letters refer to the significance between different concentrations within the same gene data. $C$. Analysis of gene expression relevant to the chondrogenic pathway, using $q R T$-PCR, under different concentrations of a CatK inhibitor, normalized to GAPDH and reference sample. There were significant increases in relative Aggrecan, Collagen $2 A$ and SOX 9 gene expressions, which were VEL0230- concentration dependent $(P<0.05)$. 

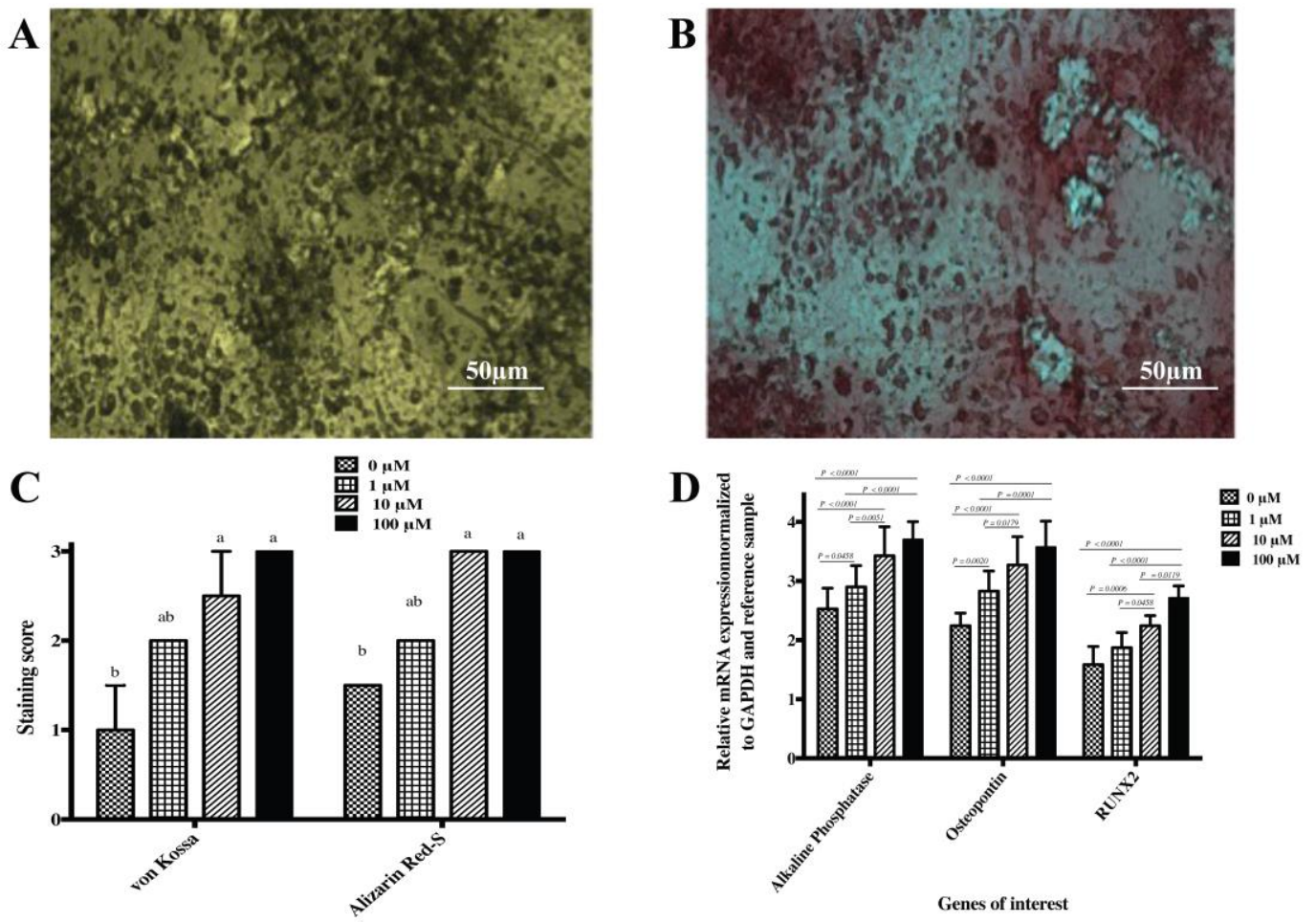

\begin{abstract}
Figure 4 Effect of CatK inhibition on osteogenic potential of equine BMD-MSCs. Cells were induced to form osteoblast by culturing in osteogenic induction medium for 28 days, followed by staining with von Kossa $(\boldsymbol{A})$ and Alizarin Red-S $(\boldsymbol{B})$. C. Median with interquartile range of von Kossa and Alizarin Red-S staining score of induced BMDMSCs; there was a significant increase due to CatK inhibition at a VEL-0230 concentration of 10 and $100 \mu M$ compared to the other concentrations (P<0.05). Different letters refer to the significance between different concentrations within the same gene data. $D$. Analysis of gene expression relevant to the osteogenic pathway, using qRT$P C R$, under different concentrations of a CatK inhibitor, normalized to GAPDH and reference sample. There were significant increases in relative alkaline phosphatase, osteopontin, and RUNX2 gene expressions, which were VEL-0230- concentration dependent $(P<0.05)$.
\end{abstract}

\section{Discussion}

For the first time, we characterized the spatial expression pattern of CatK protein in representative decalcified sections of equine weightbearing and non-weight-bearing bone. Cathepsin $\mathrm{k}$ was abundantly expressed in bone cells; osteoclasts and osteoblasts. Furthermore, we propose a novel regulatory function for CatK in bone marrow stem and progenitor cell differentiation. Besides retaining their differentiation capacities, ex vivo selected equine bone marrow stem and progenitor cells had significantly greater osteoclastogenic, chondrogenic and osteogenic differentiation potential under the effect VEL-0230, implying an inhibitory role for CatK. As hypothesized, CatK inhibition using VEL-0230 in concentrations comparable to what suppressed pro-inflammatory cytokines secretion by stimulated equine bone marrow cells in our previous study ${ }^{[9]}$, has significantly altered the differentiation potential of these cells along the three main cell lineage of the skeletal system in vitro. Owing to the close relation and tight integration between the immune and skeletal systems, multiple osteoimmunoregulatory molecules have been identified and reviewed ${ }^{[26]}$. For instance, macrophage colony-stimulating factor (M$\mathrm{CSF})$, receptor activator of nuclear factor- $\kappa \mathrm{B}(\mathrm{NF}-\kappa \mathrm{B}), \mathrm{RANKL}$, and tumor necrosis factor (TNF)-family cytokine are all examples for such molecules, which are expressed/secreted by immune cells and also regulate osteoclastogenesis. It is possible that CatK could similarly exert such regulatory roles in both immune response and skeletal system cell differentiation in vivo. Although limited evidence regarding CatK expression in immune cells such as macrophage and dendritic cells have been established so far ${ }^{[5,27]}$, possible uptake by those and other immune and differentiating progenitor cells may occur through mechanisms of endocytic recycling which may explain such osteoimmunoregulatory of CatK ${ }^{[28]}$.
All osteoclasts and a moderate number of bone lining osteoblasts stained positive for CatK indicating abundance in bone and potential for influence on bone turnover in horses. While there were no differences in CatK staining intensity and percent CatK-positive cells of total cells between tuber coxae non-weight-bearing bone and first phalanx weight-bearing bone, there was a significantly greater number of osteoclasts per weight-bearing bone surface compared to the non weight-bearing bone. This is presumably due to the fact that weight bearing is an important factor influencing bone remodelling including the osteoclastic bone resorption phase in which CatK would play an important role ${ }^{[29]}$. Cathepsin $\mathrm{K}$ is the main collagenolytic proteinase secreted by osteoclasts in the boneresorbing state ${ }^{[4]}$. Although it has long been thought that osteoclasts are the only source of CatK, CatK expression was detected in other cell types in health and disease conditions ${ }^{[11-14,16,30,31]}$. In this study, a relatively great percent of bone lining osteoblasts of healthy horses showed marked expression of CatK similar to what have been reported in human bone ${ }^{[15]}$ and did explain in part the bone resorption activity of osteoblast-like cells that have been revealed in a previous report ${ }^{[32]}$. There is a continuous need for optimization of current and future anti-resorptive medications to minimize potential adverse effect on bone formation and promote dual bone antiresorptive anabolic effect ${ }^{[33]}$. Hence, the previous findings of our study highlighted the need for more research regarding the potential effect of different CatK inhibitors on bone formation and osteoblast function.

The results of the current study revealed increased osteoclastogenic, chondrogenic and osteogenic differentiation potential of equine bone marrow derived stem and progenitor cells in contact with CatK inhibitor. The increase in the osteoclastogenic differentiation 
potential was only noted at the greatest concentration of VEL-0230 in culture media. Previous data has reported elevated levels of osteoclastogenesis in CatK-deficient mice possibly as a positive feedback of impaired bone resorption ${ }^{[34]}$. It is possible that a positive feedback loop could initiate osteoclastogenesis to compensate for the loss of CatK activity and the pause in bone resorption, the later which was not investigated in our study. While these data may suggest that CatK inhibition, as an antiresorptive therapeutic approach, may be overridden by the up-regulation of osteoclastogenesis, the clinical relevance of such outcome might be influenced by effects on the primary osteoclast progenitor cell population in vivo and also the therapeutic drug dose. In the current study, VEL-0230 was used in vitro in concentrations greater than the maximum plasma concentration achieved in vivo after drug administration in our previous studies. VEL-0230 was used in a mass concentration of 0.3 $\mu \mathrm{g} / \mathrm{ml}$ of culture media to achieve the lowest molar concentration of $1 \mu \mathrm{M}$. However, the maximum plasma concentration of VEL-0230 did not exceed $0.1 \mu \mathrm{g} / \mathrm{ml}$, achieved using an oral dose regimen of $4 \mathrm{mg} / \mathrm{kg}$ of body weight q7 days in healthy exercising horses, and by which resulted in a marked decline in bone resorption without altering the number of osteoclasts as confirmed by TRAP staining of bone biopsies and subsequent analysis ${ }^{[8,10]}$.

Cathepsin $\mathrm{K}$ inhibition in vitro significantly up-regulated the differentiation potential of BMD-MSCs towards the chondrogenic and osteogenic pathways in a VEL-0230-concentration dependent manner, implying a causal relationship. These data may provide relevance into the clinical efficacy of CatK inhibition on bone and cartilage and suggest potential anabolic effects of this particular antiresorptive approach on bone and cartilage regeneration. It has been concluded that osteoclast specific CatK deletion stimulates S1P-dependent bone formation ${ }^{[35]}$. The coupling between bone resorption and formation has been established and could account, in part, for the lack of, or decrease in, osteoblastic activity due to other anti-resorptive administration $^{[36]}$. However, our previous research using repeated VEL-0230 dosing in horses did reveal a significant increase in bone formation biomarkers ${ }^{[10]}$ and agreed with other research utilizing different CatK inhibitors, which reported maintained or increased bone formation due to therapeutic inhibition of $\mathrm{CatK}^{[37-40]}$.

Our current study provided important findings and additional data for the therapeutic inhibition of CatK, which may be relevant to the clinical use of VEL-0230 in horses. Bisphosphonates, a major antiresorptive class of drugs, have gained popularity in veterinary medicine with wide range of therapeutic applications in equine musculoskeletal disorders that are associate with increased bone resorption/inflammation ${ }^{[41]}$. However, multiple reports have elucidated the off-target effect of bisphosphonates administration and in vitro cell culture treatment on osteoclastogenesis as well as motility, viability and osteogenic differentiation of osteoblast progenitors, which in turn resulted in over suppression of bone turnover and formation, leading to osteonecrosis ${ }^{[42-45]}$.

\section{Conclusion}

We concluded that the use of CatK inhibition over other current systemic, local, or regional treatments to moderate bone resorption and inflammation in equine osseous disorders would not be anticipated to interfere with the stem and progenitor cells regenerative and differentiation capacities and may offer advantages that would require further study.

\section{Referemces}

1. Mukherjee K, Chattopadhyay N. Pharmacological inhibition of cathepsin K: A promising novel approach for postmenopausal osteoporosis therapy. Biochem. Pharmacol.2016;117:10-9.
2. Russell RGG, Tsoumpra MK, Lawson MA, Chantry AD, Ebetino FH, Pazianas M. Antiresorptives. The Duration and Safety of Osteoporosis Treatment: Springer; 2016; 17-36.

3. McDougall J, Schuelert N, Bowyer J. Cathepsin K inhibition reduces CTXII levels and joint pain in the guinea pig model of spontaneous osteoarthritis. Osteoarthr. Cartil.2010;18(10):1355-7.

4. Svelander L, Erlandsson-Harris H, Astner L, Grabowska U, Klareskog L, Lindstrom E, Hewitt E. Inhibition of cathepsin K reduces bone erosion, cartilage degradation and inflammation evoked by collagen-induced arthritis in mice. Eur. J. Pharmacol.2009;613(1):155-62.

5. Asagiri M, Hirai T, Kunigami T, Kamano S, Gober H-J, Okamoto K, Nishikawa K, Latz E, Golenbock DT, Aoki K. Cathepsin Kdependent toll-like receptor 9 signaling revealed in experimental arthritis. Science2008;319(5863):624-7.

6. Hao L, Chen J, Zhu Z, Reddy MS, Mountz JD, Chen W, Li Y-P. Odanacatib, a cathepsin K-Specific inhibitor, inhibits inflammation and bone loss caused by periodontal diseases. J. Periodontol.2015;86(8):972-83.

7. Hirai T, Kanda T, Sato K, Takaishi M, Nakajima K, Yamamoto M, Kamijima R, DiGiovanni J, Sano S. Cathepsin $\mathrm{K}$ is involved in development of psoriasis-like skin lesions through TLR-dependent Th17 activation. J. Immun. 2013;190(9):4805-11.

8. Hussein $\mathrm{H}$, Ishihara $\mathrm{A}$, Menendez $\mathrm{M}$, Bertone A. Pharmacokinetics and bone resorption evaluation of a novel Cathepsin K inhibitor (VEL-0230) in healthy adult horses. J. Vet. Pharmacol. Ther.2014;37(6):556-64.

9. Hussein H, Boyaka P, Dulin J, Bertone A. Cathepsin K inhibition renders equine bone marrow nucleated cells hypo-responsive to LPS and unmethylated CpG stimulation in vitro. Comp. Immunol. Microbiol. Infect. Dis.2016;45:40-7.

10. Hussein H, Dulin J, Smanik L, Drost W, Russell D, Wellman M, Bertone A. Repeated oral administration of a cathepsin K inhibitor significantly suppresses bone resorption in exercising horses with evidence of increased bone formation and maintained bone turnover. J Vet Pharmacol Ther. 2017;40(4):327-334.

11. Drake FH, Dodds RA, James IE, Connor JR, Debouck C, Richardson S, Lee-Rykaczewski E, Coleman L, Rieman D, Barthlow R. Cathepsin K, but not cathepsins B, L, or S, is abundantly expressed in human osteoclasts. J. Biol. Chem.1996;271(21):12511-6.

12. Hummel KM, Petrow P, Franz J, Müller-Ladner U, Aicher W, Gay $\mathrm{R}$, Brömme D, Gay S. Cysteine proteinase cathepsin K mRNA is expressed in synovium of patients with rheumatoid arthritis and is detected at sites of synovial bone destruction. J rheumat. 1998;25(10):1887-94.

13. Muir P, Schamberger GM, Manley PA, Hao Z. Localization of Cathepsin K and Tartrate - Resistant Acid Phosphatase in Synovium and Cranial Cruciate Ligament in Dogs with Cruciate Disease. Vet. Surg.2005;34(3):239-46.

14. Uusitalo H, Hiltunen A, Söderström M, Aro H, Vuorio E. Expression of cathepsins B, H, K, L, and $\mathrm{S}$ and matrix metalloproteinases 9 and 13 during chondrocyte hypertrophy and endochondral ossification in mouse fracture callus. Calcif. Tissue Int.2000;67(5):382-90.

15. Mandelin J, Hukkanen M, Li T-F, Korhonen M, Liljeström M, Sillat T, Hanemaaijer R, Salo J, Santavirta S, Konttinen YT. Human osteoblasts produce cathepsin K. Bone 2006;38(6):769-77.

16. Vinardell T, Dejica V, Poole A, Mort J, Richard H, Laverty S. Evidence to suggest that cathepsin $\mathrm{K}$ degrades articular cartilage in naturally occurring equine osteoarthritis. Osteoarthritis Cartilage 2009;17(3):375-83.

17. Gray A, Davies M, Jeffcott L. Localisation and activity of cathepsins $\mathrm{K}$ and $\mathrm{B}$ in equine osteoclasts. Res. Vet. Sci.2002;72(2):95-103.

18. Fortier LA, Potter HG, Rickey EJ, Schnabel LV, Foo LF, Chong LR, Stokol T, Cheetham J, Nixon AJ. Concentrated bone marrow aspirate improves full-thickness cartilage repair compared with microfracture in the equine model. J Bone \& Joint Surg. 2010;92(10):1927-37.

19. Godwin E, Young N, Dudhia J, Beamish I, Smith R. Implantation of bone marrow-derived mesenchymal stem cells demonstrates improved outcome in horses with overstrain injury of the superficial digital flexor tendon. Equine Vet. J.2012;44(1):25-32. 
20. Herthel DJ, editor. Enhanced suspensory ligament healing in 100 horses by stem cells and other bone marrow components. AAEP proceedings; 2001.

21. Tidball JG, Villalta SA. Regulatory interactions between muscle and the immune system during muscle regeneration. Amer. J. Physi. Reg., Integ. and Comp. Phys.2010;298(5):R1173-R87.

22. Liu Y, Wang L, Kikuiri T, Akiyama K, Chen C, Xu X, Yang R, Chen W, Wang S, Shi S. Mesenchymal stem cell-based tissue regeneration is governed by recipient $\mathrm{T}$ lymphocytes via IFN[gamma] and TNF-[alpha]. Nat. Med.2011;17(12):1594-601.

23. Mourkioti F, Rosenthal N. IGF-1, inflammation and stem cells: interactions during muscle regeneration. Trends Immunol.2005;26(10):535-42.

24. Sotiropoulou PA, Perez SA, Gritzapis AD, Baxevanis CN, Papamichail M. Interactions between human mesenchymal stem cells and natural killer cells. Stem Cells2006;24(1):74-85.

25. Bratthauer GL. The Avidin-Biotin Complex (ABC) Method and Other Avidin-Biotin Binding Methods. Immunocytochemical methods and protocols2010:257-70.

26. Takayanagi H. Osteoimmunology: shared mechanisms and crosstalk between the immune and bone systems. Nat. Rev. Immu. 2007;7(4):292-304.

27. Bühling F, Reisenauer A, Gerber A, Krüger S, Weber E, Brömme D, Roessner A, Ansorge S, Welte T, Röcken C. Cathepsin K-a marker of macrophage differentiation? J path. 2001;195(3):375-82.

28. Grant BD, Donaldson JG. Pathways and mechanisms of endocytic recycling. Nature reviews Molecular cell biology 2009;10(9):597608.

29. Vico L, Collet P, Guignandon A, Lafage-Proust M-H, Thomas T, Rehailia M, Alexandre C. Effects of long-term microgravity exposure on cancellous and cortical weight-bearing bones of cosmonauts. The Lancet2000;355(9215):1607-11.

30. Sukhova GK, Shi G-P, Simon DI, Chapman HA, Libby P. Expression of the elastolytic cathepsins $\mathrm{S}$ and $\mathrm{K}$ in human atheroma and regulation of their production in smooth muscle cells. J. Clin. Invest.1998;102(3):576.

31. Bühling F, Waldburg N, Gerber A, Häckel C, Krüger S, Reinhold D, Brömme D, Weber E, Ansorge S, Welte T. Cathepsin K expression in human lung. Cellular Peptidases in Immune Functions and Diseases 2: Springer; 2002. p. 281-6.

32. Mulari M, Qu Q, Härkönen P, Väänänen H. Osteoblast-like cells complete osteoclastic bone resorption and form new mineralized bone matrix in vitro. Calcif. Tissue Int.2004;75(3):253-61.

33. Karsdal MA, Qvist P, Christiansen C, Tanko LB. Optimising antiresorptive therapies in postmenopausal women. Drugs 2006;66(15):1909-18.

34. Kiviranta R, Morko J, Alatalo SL, NicAmhlaoibh R, Risteli J, Laitala-Leinonen $\mathrm{T}$, Vuorio E. Impaired bone resorption in cathepsin K-deficient mice is partially compensated for by enhanced osteoclastogenesis and increased expression of other proteases via an increased RANKL/OPG ratio. Bone 2005;36(1):159-72.

\section{Abbreviations}

$\begin{array}{ll}\text { CatK: } & \text { Cathepsin K } \\ \text { OC: } & \text { Osteoclast } \\ \text { TLR: } & \text { Toll like receptors } \\ \text { TRAP: } & \text { Tartrate-resistant acid phosphatase } \\ \text { qRT-PCR: } & \text { quantitative real time polymerase chain reaction } \\ \text { RANKL: } & \text { Receptor activator of nuclear factor kappa-B ligand } \\ \text { RUNX2: } & \text { Runt-related transcription factor 2 } \\ \text { TC: } & \text { Tuber coxae } \\ \text { P1: } & \text { Proximal phalanx } \\ \text { ROIs: } & \text { Regions of interest } \\ \text { BS: } & \text { Bone surface } \\ \text { BMD-MSCs: } & \text { Bone marrow derived-mesenchymal stromal cells } \\ \text { OCLs: } & \text { Osteoclast-like cells } \\ \text { Ct: } & \text { Cycle threshold } \\ \text { GAPDH: } & \text { Glyceraldehyde 3-phosphate dehydrogenase } \\ \text { SD: } & \text { Standard deviation } \\ \text { M-CSF: } & \text { Macrophage colony-stimulating factor } \\ \text { TNF: } & \text { Tumor necrosis factor }\end{array}$

35. Lotinun S, Kiviranta R, Matsubara T, Alzate JA, Neff L, Lüth A, Koskivirta I, Kleuser B, Vacher J, Vuorio E. Osteoclast-specific cathepsin $\mathrm{K}$ deletion stimulates $\mathrm{S} 1 \mathrm{P}$-dependent bone formation. The J. clin.invest. 2013;123(2):666-81.

36. Hochberg MC, Greenspan S, Wasnich RD, Miller P, Thompson DE, Ross PD. Changes in bone density and turnover explain the reductions in incidence of nonvertebral fractures that occur during treatment with antiresorptive agents. J of Clinical Endocr. \& Met. 2002;87(4):1586-92.

37. Xiang A, Kanematsu M, Kumar S, Yamashita D, Kaise T, Kikkawa H, Asano S, Kinoshita M. Changes in micro-CT 3D bone parameters reflect effects of a potent cathepsin $\mathrm{K}$ inhibitor (SB553484) on bone resorption and cortical bone formation in ovariectomized mice. Bone 2007;40(5):1231-7.

38. Jerome C, Missbach M, Gamse R. Balicatib, a cathepsin K inhibitor, stimulates periosteal bone formation in monkeys. Osteoporos. Int.2011;22(12):3001-11.

39. Pennypacker BL, Chen CM, Zheng H, Shih MS, Samadfam R, Duong LT. Inhibition of Cathepsin K Increases Modeling-Based Bone Formation, and Improves Cortical Dimension and Strength in Adult Ovariectomized Monkeys. J. Bone Miner. Res. 2014;29(8):1847-58.

40. Schaller S, Henriksen K, Sveigaard C, Heegaard AM, Hélix N, Stahlhut M, Ovejero MC, Johansen JV, Solberg H, Andersen TL. The chloride channel inhibitor NS3736 prevents bone resorption in ovariectomized rats without changing bone formation. J. Bone Miner. Res.2004;19(7):1144-53.

41. Soto SA, Barbará AC. Bisphosphonates: pharmacology and clinical approach to their use in equine osteoarticular diseases. J. Equ. Vet. Sci. 2014;34(6):727-37.

42. Bartl R, Frisch B, von Tresckow E, Bartl C. Bisphosphonates in medical practice: actions-side effects-indications-strategies: Springer Science \& Business Media; 2007.

43. Piccione M, Durgam S, Stewart M. Impact of Bisphosphonate on Osteoprogenitor Cell Differentiation. Enliven: J Stem Cells Regen Med2016;3(2):003.

44. Acil Y, Möller B, Niehoff P, Rachko K, Gassling V, Wiltfang J, Simon M. The cytotoxic effects of three different bisphosphonates in-vitro on human gingival fibroblasts, osteoblasts and osteogenic sarcoma cells. J. Cranio-Maxillofacial Surg.2012;40(8):e229-e35.

45. Hughes D, MacDonald B, Russell R, Gowen M. Inhibition of osteoclast-like cell formation by bisphosphonates in long-term cultures of human bone marrow. J. Clin. Invest.1989;83(6):1930. 
Bertone AL, et al. J Stem Cells Regen Med 2017; 13(2)

\section{Potential Conflicts of Interests}

None

\section{Acknowledgements}

The authors thank FreeStride Therapeutics, USA, for the generous supply of VEL-0230. This study was supported by the Trueman Endowment at the College of Veterinary Medicine and a PhD Scholarship for Dr. Hussein from the Egyptian Ministry of Higher Education.

* This work was presented as a poster at The 9th Annual Translational to Clinical (T2C) Regenerative Medicine Wound Care Conference, Columbus, OH, USA, May 2016.

\section{Corresponding Author}

Alicia Bertone, Address: Veterinary Medical Academic Building 1900 Coffey Rd., Room 325, Columbus, OH 43210 USA; Email: Bertone.1@ osu.edu 